\title{
Glass Phase Expelling During Liquid Phase Sintering of YSZ
}

\author{
Milton Ferreira de Souza ${ }^{\mathrm{a}}$, Dulcina Pinatti Ferreira de Souza ${ }^{\mathrm{b}}$ \\ ${ }^{a}$ Departamento de Física e Ciência de Materiais, \\ Universidade de São Paulo, \\ C.P. 369, 13.560-970 São Carlos - SP, Brazil; \\ e-mail: mfs@ifsc.sc.usp.br \\ ${ }^{\mathrm{b}}$ Departamento de Engenharia de Materiais, \\ Universidade Federal de São Carlos, \\ C.P. 676, 13.565-905 São Carlos - SP, Brazil; \\ e-mail:dulcina@power.ufscar.br
}

Received: July 2, 1998; Revised: August 20, 1998

\begin{abstract}
Expelling of the liquid phase during sintering of $\mathrm{ZrO}_{2}-6.5 \mathrm{~mol} \% \mathrm{Y}_{2} \mathrm{O}_{3}-0.5 \mathrm{~mol} \% \mathrm{Pr}_{2} \mathrm{O}_{3}$ ceramic was observed as a result of grain coarsening. $\mathrm{ZrO}_{2}-7.0 \mathrm{~mol} \% \mathrm{Y}_{2} \mathrm{O}_{3}$ samples, without $\mathrm{Pr}_{2} \mathrm{O}_{3}$ addition, do not show this effect under the same sintering conditions. The expelling process is caused by surface tension forces and attracting van der Waals forces between the grains, coupled with the existence of two glass phases on the grain boundaries. The amount of expelled glass phase increases with grain growth, but saturates above $16 \mu \mathrm{m}$ average grain size.
\end{abstract}

Keywords: liquid phase sintering, glass expelling, zirconia

\section{Introduction}

Glass phase expelling during ceramic liquid phase sintering at atmospheric pressure is an uncommon phenomenon. Expelling must be distinguished from superficial grain wetting, a very common process. Large liquid masses are involved in the expelling process, resulting in the formation of glass drops of up to $30.0 \mu \mathrm{m}$ on the external ceramic surface. Ceramic liquid phase sintering was discussed for the first time by Kingery ${ }^{1}$. An analysis of the capillary forces during liquid phase sintering was given by Cahn and Heady $^{2}$. Expelling of the liquid phase from the grain boundary under the action of external forces was discussed by Lange $^{3}$. Liquid phase expelling during ceramic sintering under atmospheric pressure, however, has not been treated in the literature, to our knowledge.

This paper discusses the conditions necessary for glass expelling in order to understand the experimental results on silicate liquid phase sintering of Yttria stabilised Zirconia with a small addition of Praseodymia.

\subsection{Theoretical background}

For the purpose of clear and comprehensive considerations, a short review of surface tension and the resulting forces on ceramic grains is presented. Let us consider a wetting liquid phase inside a solid tube, as indicated in Figs. $1 \mathrm{~A}$ and 1B. The difference in pressure between the two faces of the surface exposed to air is expressed by

$$
\mathrm{P}-\mathrm{P}_{0}=\gamma_{\mathrm{LV}} \cos \theta\left(\frac{1}{\mathrm{r}_{1}}+\frac{1}{\mathrm{r}_{2}}\right)
$$

where $r_{1}$ and $r_{2}$ are the principal curvature radii of the liquid-vapour interface and $\gamma_{\mathrm{LV}}$ the liquid-vapour interfacial tension ${ }^{4}$. Another representation for the interfacial tension is to consider the associated forces, as illustrated in Fig. 1C, where the contact between a solid, S, and a liquid, $\mathrm{L}$, and their vapours, $\mathrm{V}$, is shown. In this case $\gamma_{\mathrm{SL}}, \gamma_{\mathrm{LV}}$, and $\gamma_{\mathrm{SV}}$ are the interfacial tensions between solid-liquid, liquid-vapour, and solid-vapour, respectively, in a vector representation.

Let us take the grain boundary of a polycrystalline liquid phase sintered material as being the region of thickness $\delta_{\mathrm{gb}}$ between two plane surfaces of length d, and width 1 , as shown in Fig. 2. For sintering to proceed, it is necessary for the liquid phase to wet the grain surface. The liquid volume between the plates is given approximately by $\mathrm{V}_{\mathrm{L}}=\delta_{\mathrm{gb}} \mathrm{d}$ l. Grain weight will be not be taken into account 

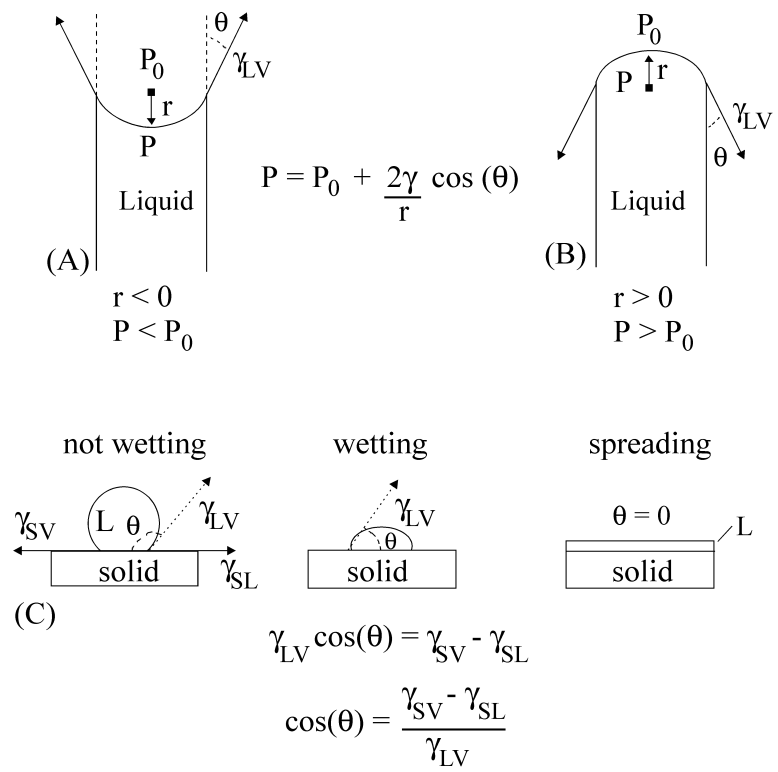

Figure 1. Liquid-solid-gas interface: A- liquid internal pressure, $P$, smaller than atmospheric pressure, $\mathrm{P} 0 ; \mathrm{B}-\mathrm{P}>\mathrm{P}_{0}$ turning positive the curvature radius; $\mathrm{C}$ - liquid behavior on a solid surface for several wetting angles.
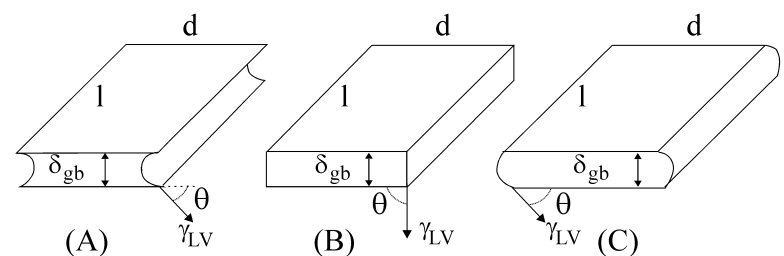

Figure 2. Two plane plates simulating two grains with a grain boundary liquid phase. A- Plates are kept apart by applied external forces; B- null external forces; C- compressing external forces.

in this paper, but internal forces between grains and externally applied forces will be considered.

In applying Eq. 1 to Fig. 2A, the principal curvature radii are $-r$ and $\infty$. The maximum absolute value for $r$ is $\mathrm{r}=\delta_{\mathrm{gb}} / 2$ corresponding to $\cos \theta=1$. Therefore, according to Eq. 1, although the pressure decreases in the liquid relative to $P_{0}$, surface tension forces will cause the plates to approach each other. It is a well-known fact that this process occurs during the first stage of ceramic liquid phase sintering. The grains get closer to each other until the repulsive forces between the two grains equilibrate the difference in pressure ${ }^{1}$. In the absence of other forces, the grain boundary thickness, $\delta_{\mathrm{gb}}$, will become thinner and full of the liquid phase. In this situation, the contact angle, $\pi$, will become $\theta=\pi / 2$, reaching equilibrium because the internal and external pressures become equal, as shown in Fig. 2B. Figure 2C shows the opposite situation: the inter- nal pressure between the plates is larger than the external pressure. According to Eq. 1,

$$
P_{C}=P_{o}+\gamma_{L V} \frac{\cos \theta}{r_{c}}
$$

In this case, the capillary forces would pull apart the plates. The maximum pressure between the plates is given by

$$
\mathrm{P}_{\mathrm{C} \max }=\mathrm{P}_{\mathrm{o}}+\frac{2 \gamma_{\mathrm{LV}}}{\delta_{\mathrm{gb}}}
$$

Therefore, the maximum force that can be applied to the plates, other than the isostatic external pressure, is limited to the over pressure in the liquid phase, as expressed by Eq. 3. Without the application of forces on the plates, the capillary forces will separate the grains until $\theta$ becomes $\theta=\pi / 2$, the equilibrium configuration.

Let us now consider grain coarsening during the liquid phase sintering process of a ceramic body under atmospheric pressure. Grain growth results in a decrease of the total grain surface area, causing an increase of the grain boundary liquid phase thickness. Under the imposed conditions shown in Fig. 2C, considering Eq. 3 and the absence of forces other than the capillary ones, no liquid phase can be expelled because the capillary forces change the configuration to equilibrium, as shown in Fig. 2B. Penetration of ceramic bodies by liquid glass phase ${ }^{5,6}$ is a similar case. The liquid phase can penetrate the sintered ceramic body, increasing the ceramic body's volume by as much as $40 \%$. Large amounts of liquid can penetrate the solid body if the liquid phase fully separates the grains, the ceramic grains remaining in suspension in the liquid glass and the solid shape tending to be spherical.

In the above-described situations, it has been assumed that ceramic grains are fully separated by the liquid phase. This may, however, not be the case if attractive forces between the grains are taken in account. Attractive van der Waals forces and repulsive forces resulting from the distorted chemical bonds in the solid-liquid interface determine the limiting thickness of the glass phase in the grain boundary. According to Clarke ${ }^{7}$, the equilibrium between these forces is reached when the thickness is near $2.0 \mathrm{~nm}$. Therefore, full penetration implies that the internal pressure must be sufficient to overcome these attractive forces. It may be that parts of the grain boundary are thin while other parts are thicker in ceramic bodies sintered by liquid phase sintering. Attractive forces allow the internal pressure during grain growth to increase, stabilising the situation shown in Fig. 2C. If the attractive forces are sufficiently strong to allow the internal pressure to be higher than the pressure given by Eq. 3, then the wetting liquid glass phase will be expelled from the ceramic and will spread over its external surface. At intermediate stages, only part of the grain 
boundary will be expanded to make room for the liquid glass phase, producing a grain boundary microstructure showing thick and thin regions.

\section{Experimental}

Ceramic discs, whose composition is given in Table 1, were isostatically pressed at $200 \mathrm{MPa}$ and sintered at 1610 and $1550{ }^{\circ} \mathrm{C}$ for several different time intervals inside alumina crucibles, with the plane face in the vertical position. A reference composition, free of the rare earth doping, was also prepared where the $\operatorname{Pr}_{2} \mathrm{O}_{3}$ concentration in Table 1 was substituted by $\mathrm{Y}_{2} \mathrm{O}_{3}$. Cooling rates were of $1000^{\circ} \mathrm{C} / \mathrm{h}$. Microstructural observations were done using SEM (Zeiss 9600, Germany) and optical microscopy (Leica DMRX, Germany) on both polished and unpolished samples. Grain size was measured by the interception method along several directions in the micrographs. Quantitative EDS analysis (Link Analytical QX 2000, England) of the glass phases and ceramic grains are shown in Table 2. The data presented in this table represents the average of ten measurements in different parts of the sample. The mass of expelled glass was measured as follows. The sintered ceramic discs were weighed, observed under the optical microscope and carefully sanded on a 600 grit silicon carbide emery paper. This sequence was repeated until the glass drops on the surface of the disc surface disappeared. A second process was used on a few samples for purposes of control. The number of large glass droplets was counted and their average diameter and height measured with the help of sensitive thickness measuring equipment for thin films (Talistep, England). The maximum observed height was $35.0 \mu \mathrm{m}$ and the contact angle of the glass droplets with the ceramic

Table 1. Chemical compositions of the samples (mol\%).

\begin{tabular}{lllllll}
\hline $\mathrm{ZrO}_{2}$ & $\mathrm{Y}_{2} \mathrm{O}_{3}$ & $\mathrm{Pr}_{2} \mathrm{O}_{3}$ & $\mathrm{Al}_{2} \mathrm{O}_{3}$ & $\mathrm{TiO}_{2}$ & $\mathrm{CaO}$ & $\mathrm{SiO}_{2}$ \\
\hline 89,5 & 6,5 & 0,5 & 0,5 & 0,12 & 0,12 & 2,5 \\
\hline
\end{tabular}

Table 2. Average compositions of grains and glass phase ( $\mathrm{mol} \%)$.

\begin{tabular}{lccc}
\hline & $\begin{array}{c}\text { Expelled glass } \\
\left(\mathrm{G}_{1}\right)\end{array}$ & $\begin{array}{c}\text { Grain boundary } \\
\text { glass }\left(\mathrm{G}_{2}\right)\end{array}$ & Grain \\
\hline $\mathrm{Al}_{2} \mathrm{O}_{3}$ & 9,0 & 9,0 & Trace \\
$\mathrm{CaO}$ & 1,8 & 2,1 & Trace \\
$\mathrm{SiO}_{2}$ & 74,6 & 69,0 & 0,5 \\
$\mathrm{ZrO}_{2}$ & 2,0 & 7,2 & 92,4 \\
$\mathrm{TiO}_{2}$ & 1,0 & 1,1 & Trace \\
$\mathrm{Y}_{2} \mathrm{O}_{3}$ & 5,5 & 6,7 & 6,5 \\
$\mathrm{Pr}_{2} \mathrm{O}_{3}$ & 6,0 & 5,0 & 0,3 \\
\hline
\end{tabular}

surface was $25^{\circ}$. The rounded shape of the upper part of the glass surface was observed in an inverted optical microscope through circular Newton rings. Sample densities were measured by the water immersion technique. The infiltration experiments were done as follows. A small pellet with G1 composition was placed on the surface of an already sintered ceramic pellet, infiltrated at $1610^{\circ} \mathrm{C}$ for $6.0 \mathrm{~h}$ and cooled at $1000{ }^{\circ} \mathrm{C} / \mathrm{h}$ rate. The pellets were cut in the transversal direction by a diamond saw and polished.

\section{Results}

The density of sintered discs was found to be $5.7 \mathrm{~g} / \mathrm{cm}^{3}$, with $1 \%$ standard deviation during the entire sintering time of 0.5 to $16.0 \mathrm{~h}$. The expelled glass phase around the grain boundaries of a ceramic disc sintered at $1550{ }^{\circ} \mathrm{C}$ is clearly shown in the micrograph of Fig. 3. Spreading is low when compared with sintering at higher temperatures, probably due to the high viscosity of the expelled glass. Figure 4 shows the optical micrograph of the expelled glass phase

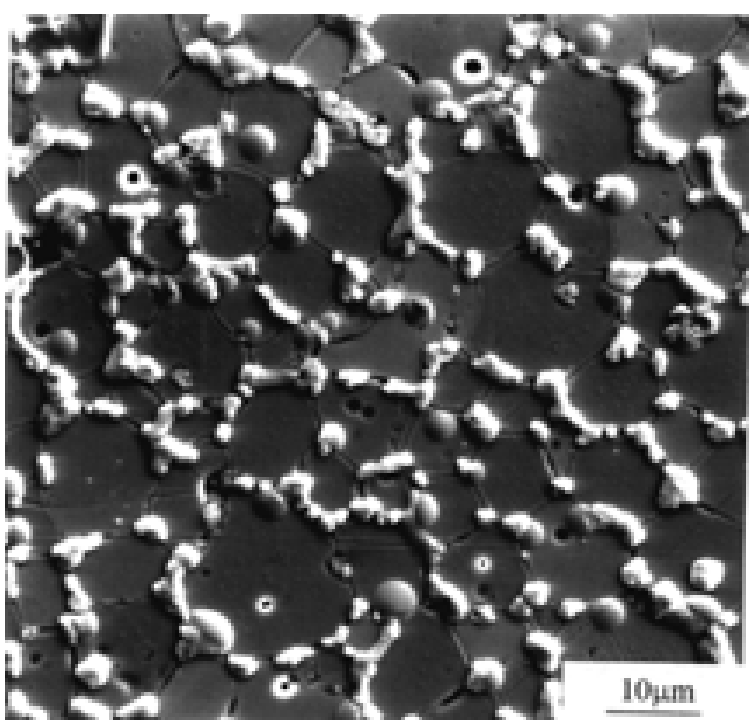

Figure 3. SEM micrograph of expelled glass phase on YSZ ceramic after $4.0 \mathrm{~h}$ of sintering at $1550^{\circ} \mathrm{C}$.

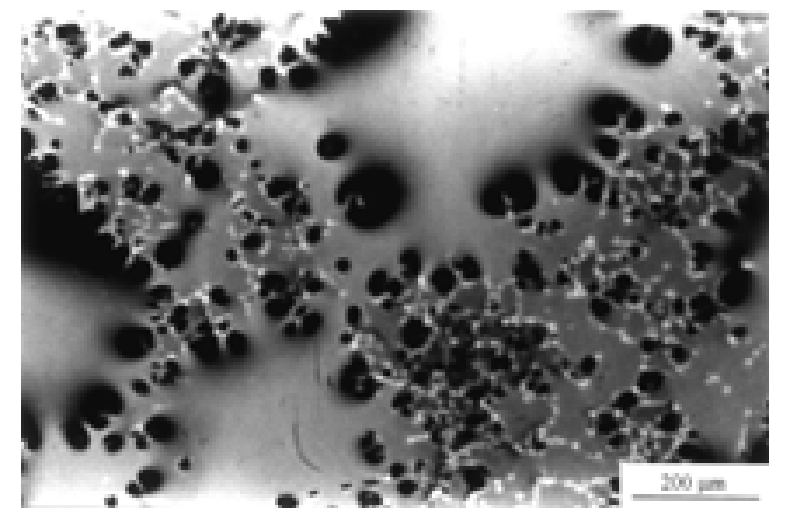

Figure 4. Optical micrograph of expelled glass phase on YSZ ceramic after $8.0 \mathrm{~h}$ of sintering at $1610^{\circ} \mathrm{C}$. 
on the surface of a ceramic disc sintered at $1610{ }^{\circ} \mathrm{C}$ during $8.0 \mathrm{~h}$. In this case, the expelled glass coalesces into large glass droplets owing to its lower viscosity. It does not, however, spread over the ceramic surface. The reference sample, free of Praseodymium, and sintered at $1610{ }^{\circ} \mathrm{C}$ for $16.0 \mathrm{~h}$ did not show an expelled glass phase. Two glass phases are present in the ceramic discs with different compositions: the one that remains inside the grain boundaries and the expelled phase, G2 and G1, respectively. Table 2 shows the composition of these two phases as well as that of the ceramic grains. The G2 phase and the EDS grain analysis were done after polishing and thermal etching of the ceramic discs, while the G1 phase was analysed in the discs after sintering without polishing.

Although EDS analyses were done on the thicker part of the G1 droplets, its measured zirconium concentration may be excessive due to the ceramic substrate's XR emission. Table 2 shows that Praseodymium is strongly segregated to the liquid phase while the Yttrium segregation coefficient is close to 1.0, with almost no segregation. The G2 phase composition has a higher zirconia and lower silica

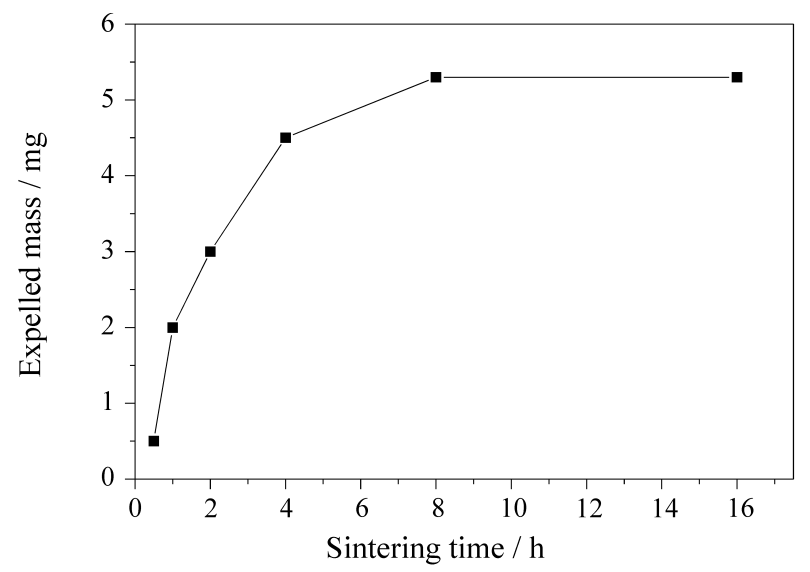

Figure 5. Expelled glass phase mass for several sintering times at $1610^{\circ} \mathrm{C}$

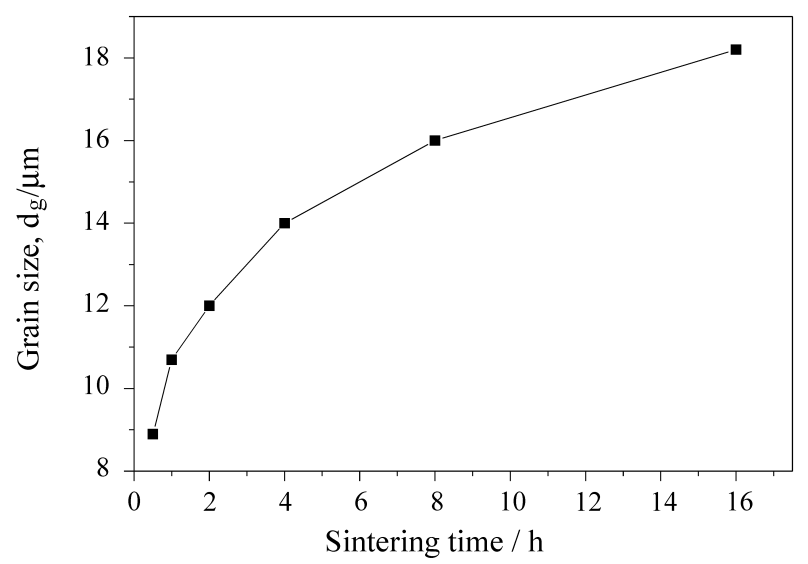

Figure 6. Grain size growth dependence with sintering time at $1610^{\circ} \mathrm{C}$. concentration than the $\mathrm{G} 1$ phase. The grain boundary glass composition was also analysed in the transversal plane of the pellet and the same G2 composition was found. Figure 5 shows the expelled mass for several sintering times. Maximum deviation on the expelled mass was $15 \%$.

Expelled mass increases with the sintering time with saturation for times over $8.0 \mathrm{~h}$. Average grain size was measured with a standard deviation of $10 \%$ and its growth with sintering time is shown in Fig. 6.

The kinetic of grain growth agrees well with the characteristic of grain coarsening in liquid phase sintering, Eq. 4:

$$
\mathrm{D}^{3}-\mathrm{D}_{\mathrm{o}}^{3}=\mathrm{Kt}
$$

where $\mathrm{D}$ is the average grain size, $\mathrm{t}$ is the sintering time and $\mathrm{K}$ a constant ${ }^{1}$. The microstructures of the ceramic discs sintered at $1610{ }^{\circ} \mathrm{C}$ for $16.0 \mathrm{~h}$ and polished are shown in Fig. 7. Two types of microstructures are shown. One of these - the one most frequently found in the discs - has its grain boundary characterised by very thin and thick regions, while in the other the grain boundary has the same thickness between two triple points. Very few pores were found, and all of them were glass filled.

Figure 8 shows the correlation between the expelled mass and the inverse of grain size. As the inverse of average grain size correlates with the grain size, this figure shows that glass expelling depends on the internal pressure generated by the decrease of the internal area for the glass phase. Figure 9 is a micrograph of a transversal section of a ceramic disc, where an excess of glass of G1 phase was infiltrated at $1610{ }^{\circ} \mathrm{C}$ during $4.0 \mathrm{~h}$, in order to thicken the glass phase around the ceramic grains and facilitate observation of the glass phase separation. Small droplets of a

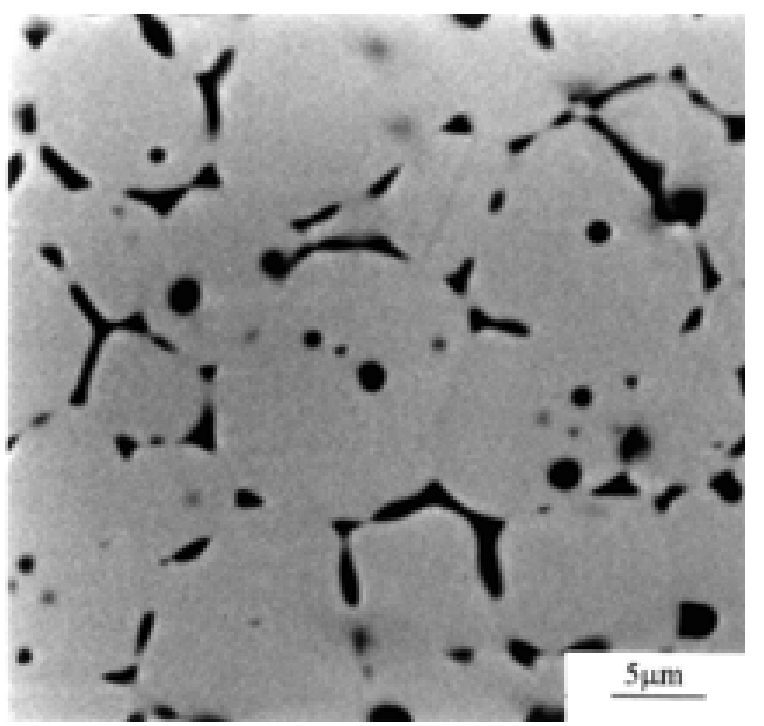

Figure 7. Characteristic glass phase distribution in the grain boundaries after sintering at $1610^{\circ} \mathrm{C}$ Thin and thick regions are observed. 


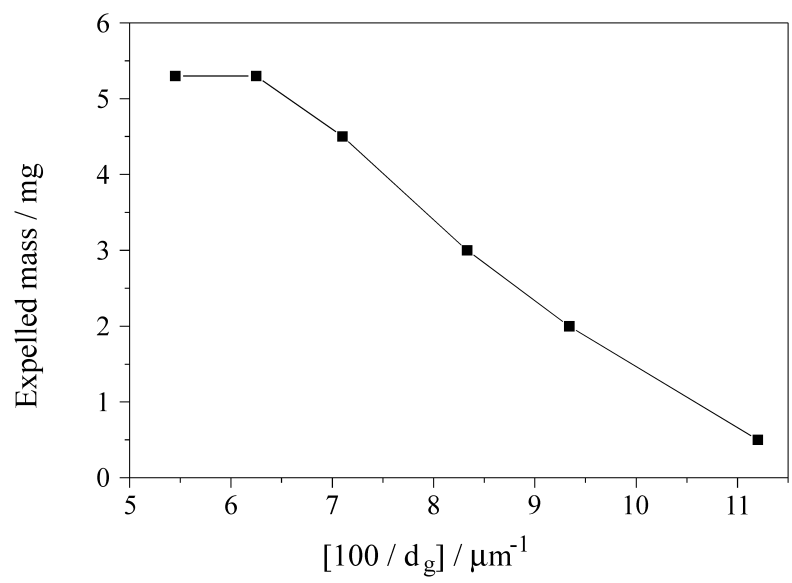

Figure 8. Dependence between the expelled glass mass and grain size.

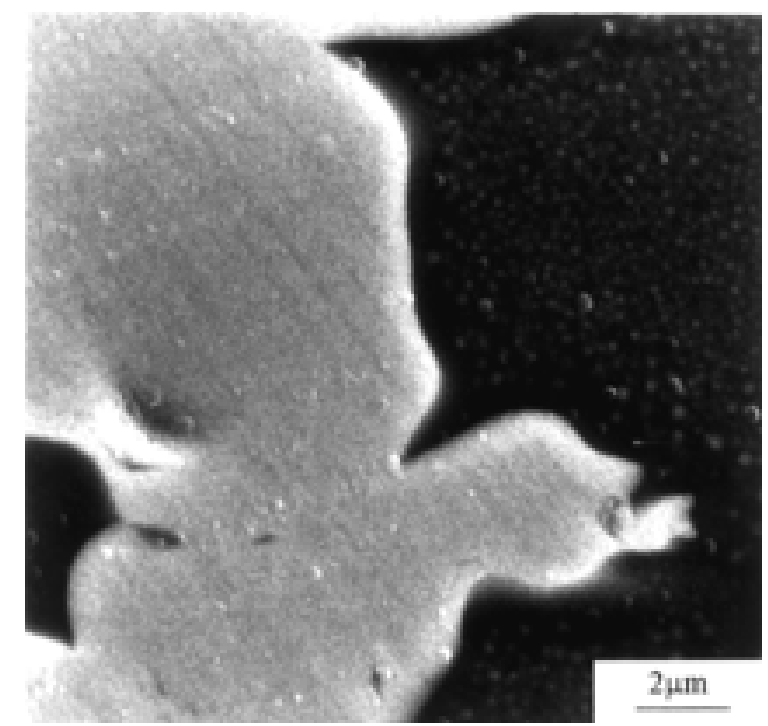

Figure 9. Transversal cut on the glass infiltrated region in zirconia plate showing glass phase separation (small spheres in the black regions) done at $1610{ }^{\circ} \mathrm{C}$ by $\mathrm{G} 1$ glass composition.

second phase can be seen in the glass phase, the darker part of the micrograph.

\section{Discussion}

The results of the previous section clearly illustrate the expelling of a glass phase, $\mathrm{G}_{1}$, whose composition is different from the other glass phase, G2, that wets the ceramic grains. Expelling through the grain boundaries that intercept the ceramic disc surface generate glass droplets that further coalesce into larger droplets but do not spread onto the ceramic grains where their contact angle is $25^{\circ}$. The infiltration experiment was done to verify that at least two glass phases were present in the grain boundaries, showing the presence of spherical droplets in the glass phase. The presence of two glass phases in the grain boundaries can therefore be taken for granted.
According to Mendelson ${ }^{8}$, the total surface grain area, $\mathrm{S}_{\mathrm{g}}$, relates to the average grain size, $\mathrm{D}_{\mathrm{g}}$, a

$$
\mathrm{S}_{\mathrm{g}}=\frac{\mathrm{C}}{\mathrm{D}_{\mathrm{g}}}
$$

where $\mathrm{C}$ is a constant that depends on grain shape and average grain size distribution. Thus, considering the linear relation between the expelled mass, $\mathrm{M}_{\mathrm{ex}}$, with the inverse of the average grain size, $D_{g}$, shown in Fig. 8, the following relation holds true

$$
\mathrm{M}_{\mathrm{ex}}=\mathrm{K}\left(\mathrm{S}_{\mathrm{go}}-\mathrm{S}_{\mathrm{g}}\right)
$$

where $S_{g_{0}}$ is the total grain surface area when the expelling process starts, and $\mathrm{K}$ the proportionality constant. Assuming the volume of sintering liquid phase is constant, the internal pressure in the sintering disc should increase if constraints are present between the grains, or the grain boundary thickness should increase to alleviate the internal pressure completely if only capillary forces are present. It is, therefore, necessary to know if internal constraints are present.

Figure 7 shows that the grain boundary is characterised by thick and thin regions. We assume that thin regions have thickness close to $2.0 \mathrm{~nm}$, as calculated by Clarke ${ }^{7}$, for the equilibrium thickness of a glassy phase between two ceramic grains. Two experimental results are presented to support this assumption. First, the strong electrical conductivity shown by these samples resulting from percolation through the thin grain boundary regions ${ }^{9}$. Second, the findings of Godickmeier et al. ${ }^{10}$ for the grain boundary thickness of silicate sintered T3YZ. The grain boundary thickness found by these authors is very close to $2.0 \mathrm{~nm}$. Therefore, the thin grain boundaries shown in Fig. 7 are regions where the attractive forces are still present because insufficient pressure was applied to pull the grains apart. Glass expelling will be analysed taking into account these forces between grains.

The internal pressure of ceramic discs generated by the decrease in the total grain surface area can become relaxed by glass expelling if there are internal constraints such as the attractive forces between grains. There are two ways for glass expelling to occur. The first case occurs when the internally developed pressure is larger than the maximum given by Eq. 3. The other possibility is expelling of a glass phase, such as the G1 phase, unrelated to the interfacial tension between the wetting glass phase and the ceramic grains. In the latter case, the G1 phase will move out of the ceramic body under the gradient pressure generated by the grain growth, the pressure increasing from the surface to the central part of the body.

The results of our experiments can be summarised as follows: a) attractive forces between the grains are present; b) decrease of the internal total grain area maintains a linear 
relation with the expelled mass; c) glass phase separates in the glass inside the grain boundaries. From these results it can be concluded that G1 glass expelling results from alleviation of the internal pressure. The increase of internal pressure is a result of the reduction of the total grain surface area and the attractive van der Waals forces between the grains. This increase of internal pressure is lower than the maximum allowed by Eq. 3 because no detectable amount of G2 phase was found on the ceramic grains' external surface. Another independent way for pressure relaxation would be partial grain separation in the thin regions. According to this hypothesis, average grain boundary thickness would increase and grain boundary conductivity would decrease, contrary to our earlier findings ${ }^{9}$. Therefore, G1 phase expelling was the only relaxation process for alleviation of the internal pressure, and also the pressure increase originating from grain growth was not sufficient to overcome the attractive forces among grains.

Praseodymium and Yttrium oxide additions to the glass phase properties contribute to the glass expelling process. It has been found by Kohli et al. ${ }^{11}$ that rare earth and Yttrium aluminosilicate glasses contain a broad distribution of non-bridging oxygens, NBO, which increase in concentration with increasing concentrations of rare earth and Yttrium oxide concentrations. The presence of NBO must contribute to lower the viscosity of G1 and G2 phases, but the G1 phase is expected to have an even lower viscosity due to its much lower $\mathrm{ZrO}_{2}$ concentration. Although Kohli et al. have found that all rare earth oxides produce NBO in aluminosilicate glass, the expelling process depends on the rare earth's ionic radius. Large ionic radius ions show a high segregation relative to the zirconia grains, increasing their concentration in the glass phases. The same behaviour was found for other large ionic radius ions, $\mathrm{Nd}^{+3}$, $\mathrm{Ca}^{+2}, \mathrm{Sm}^{+3}$, but not for smaller ions as $\mathrm{Er}^{+3}, \mathrm{Yb}^{+3}, \mathrm{Y}^{+3}$ and $\mathrm{Gd}^{+312}$.

\section{Conclusions}

The otherwise impossible glass phase expelling observed during grain coarsening in liquid phase sintered $\mathrm{ZrO}_{2}-6.5 \mathrm{~mol} \% \mathrm{Y}_{2} \mathrm{O}_{3}-0.5 \mathrm{~mol} \% \mathrm{Pr}_{2} \mathrm{O}_{3}$ ceramic is possible due to glass phase separation of the sintering liquid. The necessary conditions for increased internal pressure was found to be the grain growth and the attractive van der Waals forces between the grains. However, the internal pressure increase is limited by expelling of part of the glass phase. For glass expelling to be in accordance with the theoretical model the presence of two glass phases is nec- essary: one that fully wets the grains and the other, which will be expelled, with higher interfacial tension with the grains, as was found experimentally. The main difference in composition between these two phases is in the $\mathrm{ZrO}_{2}$ concentration. However, the presence of $\mathrm{Pr}_{2} \mathrm{O}_{3}$ in the glass composition was found to be necessary because the ceramic sample composition $\mathrm{ZrO}_{2}-7.0$ mol\% $\mathrm{Y}_{2} \mathrm{O}_{3}$ did not show glass expelling during sintering.

\section{Acknowledgments}

The financial support from FAPESP is gratefully acknowledged. The authors also express their appreciation to Professor Rolo for his help with the optical micrographs and to W.L.M. Esteves for the stimulating discussions.

\section{References}

1. Kingery, W.D. J. Appl. Phys., v. 30, n. 3, p. 301-306, 1959.

2. Cahn, J.W.; Heady, R.B. J. Am. Ceram. Soc., v. 53, n.7, p. 406-409, 1970.

3. Lange, F.F. J. Am. Ceram. Soc., v. 65, n. 2, p. C-23, 1982.

4. Chiang, Y.M.; Birnie III, D.; Kingery, W.D. Physical Ceramics, Principles for Ceramic Science and Engineering, John Wiley \& Sons, New York, 1997.

5. Marion, J.E.; Hsueh, C.H.; Evans, A.G. J. Am. Ceram. Soc., v. 70, n. 10, p. 708-713, 1987.

6. Flaitz, P.L.; Pask, J.A. J. Am. Ceram. Soc., v.70, n. 7, p. 449-455, 1987.

7. Clarke, D.R. J. Am. Ceram. Soc., v. 70, n. 1, p. 15-22, 1987.

8. Mendelson, M.I. J. Am. Ceram. Soc., v. 52, n. 8, p. 443-446, 1969.

9. De Souza, M.F.; De Souza, D.P.F. submitted to the $J$. Mater. Sci.

10. Godickmeier, M.; Michel, B.; Oliukas, A.; Bohac, P.; Sasaki, K.; Glaucker, L.; Heinrich, H.; Schwander, P.; Kostorz, G.; Hofman, H.; Frei, O. J. Mater. Res., v. 9, p. 1228-1240, 1994.

11. Kohli, J.T.; Condrate, R.A.; Shelby, Snr \& J.E. Phys. Chem. Glasses v. 34, n. 3, p. 81-87, 1993.

12. Canova, I.C. Liquid Phase Composition Effect on the Sintering and Electrical Conductivity of Zirconia-Yttria, M.S. Thesis, Dept. de Engenharia de Materiais, Universidade Federal de São Carlos - SP, Brazil, 1998. 